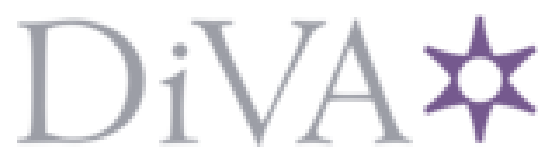

http://www.diva-portal.org

\title{
Preprint
}

This is the submitted version of a paper published in Acta Oto-Laryngologica.

Citation for the original published paper (version of record):

Edin, F., Liu, W., Li, H., Atturo, F., Magnusson, P. et al. (2014)

3-D gel culture and time-lapse video microscopy of the human vestibular nerve.

Acta Oto-Laryngologica, 134(12): 1211-1218

http://dx.doi.org/10.3109/00016489.2014.946536

Access to the published version may require subscription.

N.B. When citing this work, cite the original published paper.

Permanent link to this version:

http://urn.kb.se/resolve?urn=urn:nbn:se:uu:diva-2395 18 
3-D culture of Human Vestibular Nerve

\section{3-D gel Culture and Time Lapse Video Microscopy of the Human Vestibular} Nerve

\author{
Fredrik Edin ${ }^{1, *}$, Wei Liu ${ }^{1}$, Hao $\mathrm{Li}^{1}$, Francesca Atturo ${ }^{1,3}$ Peetra U. Magnusson ${ }^{2}$ and \\ Helge Rask-Andersen ${ }^{1}$
}

Fredrik Edin, M.Sc., Department of Surgical Sciences, Head and Neck Surgery, section of Otolaryngology, Uppsala University Hospital, SE-751 85 Uppsala, Sweden, Phone: +46-18-6115458, Fax: +46-18-500979, E-mail:

fredrik.edin@surgsci.uu.se

Wei Liu, MD. PhD, Department of Surgical Sciences, Head and Neck Surgery, section of Otolaryngology, Uppsala University Hospital, Departments of Otolaryngology, Uppsala University Hospital, SE-751 85, Uppsala, Sweden. Email:lwoo24@gmail.com

Hao Li, PhD, Department of Surgical Sciences, Head and Neck Surgery, section of Otolaryngology, Uppsala University Hospital, Departments of Otolaryngology, Uppsala University Hospital, SE-751 85, Uppsala, Sweden. Email:hao.li@surgsci.uu.se

Peetra U. Magnusson PhD, Associate Professor, ${ }^{2}$ Department of Immunology, Genetics and Pathology, Clinical Immunology, Dag Hammarskölds väg 20, 75237 Uppsala. E-mail: peetra.magnusson@igp.uu.se

Francesca Atturo, MD, ${ }^{3}$ Department of Neurology, Mental Health and Sensory Organs, Otorhinolaryngologic Unit, Medicine and Psychology, Sapienza, Rome, Italy and Surgical Sciences, Section of Otolaryngology, Uppsala University Hospital, SE-751 85,Uppsala,Sweden.E-mail: atturo@libero.it

Helge Rask-Andersen, MD, PhD, Professor, Department of Surgical Sciences, Head and Neck Surgery, section of Otolaryngology, Uppsala University Hospital, ${ }^{1}$ Departments of Otolaryngology, Uppsala University Hospital, SE751 85, Uppsala, Sweden.E-mail: helge.rask-andersen@akademiska.se

Corresponding author: Fredrik Edin, Department of Surgical Sciences, Section of Otolaryngology, Uppsala University Hospital, SE-751 85 Uppsala, Sweden, Phone: +46-18-6115458, Fax: +46-18-500979, E-mail: fredrik.edin@surgsci.uu.se

Acknowledgements: We gratefully acknowledge the help of Associate Professor Nils Hailer (Uppsala University) providing us with valuable animal material. This study was supported by ALF grants from Uppsala University Hospital and Uppsala University and by the Foundation “Tysta Skolan”, Swedish Deafness Foundation (HRF), the 


\section{3-D culture of Human Vestibular Nerve}

29 Swedish Research Council (A0290401, A0290402) and kind private donations from Börje Runögård, Sweden.. Our

30 research is part of the European Community 7th Framework Programme on Research, Technological Development

31 and Demonstration EU-FP7-NMP-2011-Small-5 "NANOCI", Project no: 281056.

\section{Abstract}

33 Conclusions: Human inner ear neurons can be grown in 3-D in a gel and documented with time-

34 lapse video microscopy (TLVM). The technique is valuable for experimental investigations of

35 human inner ear neuron signaling and regeneration.

36 Objectives: To establish a new in vitro model to study human inner ear nerve signaling and

37 regeneration.

38 Method: Human superior vestibular ganglion was harvested during translabyrinthine surgery for

39 removal of Vestibular Schwannoma. After dissection tissue explants were embedded and cultured

40 in a laminin-based 3-D matrix (Matrigel ${ }^{\mathrm{TM}}$ ). 3-D growth cone (GC) expansion was analyzed

41 using TLVM. Neural marker expression was appraised using immunocytochemistry with

42 fluorescence- and laser confocal microscopy.

43 Results: Tissue explants from adult human superior vestibular ganglion could be cultured in 3-D

44 in a gel indicating an innate potential for regeneration. Expanding growth cones were found to

45 expand dynamically in the gel. Growth cone expansion and axonal Schwann cell alignment was

46 documented using TLVM. Neurons were identified morphologically and through

47 immunohistochemical staining. 
48 Abbreviations:

49 BDNF Brain-Derived Neurotrophic Factor

50 CI Cochlear Implant

51 GC Growth cone

52 GDNF Glial cell line-Derived Neurotrophic Factor

53 GFAP Glial Fibrillary Acidic Protein

$54 \quad$ hNPC Human neural progenitor cells

$55 \quad$ NT-3 Neurotrophin-3

56 SG Spiral Ganglion

57 SVG Superior Vestibular Ganglion (Scarpa's Ganglion)

58 TLVM Time-lapse video microscopy 
59

60

61

62

63

64

65

66

67

68

\section{Introduction}

Cochlear implants (CI) have now been used for more than 50 years to treat patients with profound hearing loss with increasing success (1). More than a quarter of a million deaf people have benefitted from the treatment. The auditory nerve is stimulated electrically by-passing the sensory mechanoreceptors. Despite excellent results, particularly in deaf-born children, results are still varying (2). A problem may be the anatomical gap between the electrode and individual neurons. Minimizing or closing this gap could lead to better frequency resolution and reduced battery consumption. To regenerate or re-direct axons to electrodes requires a guidance medium inside the cochlear fluid space $(3,4)$.

Adult human cochlear neurons have been cultured in 2-D (5) but studies on human material are rare. 3-D inner ear nerve cultures have previously been established using material from experimental animals (6-8), however, to our knowledge no 3-D cultures of adult human inner ear neurons have yet been achieved. Such studies may invaluable to assess clinically significant interspecies differences $(9,10)$ and to better understand nerve guidance properties, regeneration and effects of electric stimulation in man.

Studies of human cochlear nerves are difficult to perform due to limited access to vital spiral ganglion (SG) tissue encased in hard bone. Here, we present a technique to harvest the human superior vestibular ganglion (Scarpa's ganglion, SVG) during translabyrinthine removal of vestibular schwannoma (VS). These cells are located in the internal acoustic meatus and more easily available. Both vestibular and cochlear nerves belong to the eighth cranial nerve delaminated from the otic placode (10) although developmentally differing in response to certain neurotrophins (11).

In this paper adult human SVG were successfully cultured as explants in a 3-D matrix in parallel with human progenitor nerve cell culture and animal experiments to establish a new source of human material for in vitro studies on the auditory system. 
84

85

86

87

88

89

90

91

92

93

94

95

96

97

98

99

100

101

102

103

104

105

106

107

108

109

110

111

112

\section{Materials and Methods}

Media composition

Complete 1:1 DMEM:F12 media (Gibco, Paisley, Scotland) contains N2 (1x, Gibco) and B27 supplements (1x, Gibco) as well as $1 \mathrm{mM}$ of L-glutamine (Gibco), $50 \mathrm{ng} / \mathrm{mL}$ heparan sulfate (Sigma Aldrich, Seelze, Germany) and $0.04 \%$ gentamicine (Gibco). 20 ng/mL epidermal growth factor (EGF, R\&D Systems, Minneapolis, USA), $10 \mathrm{ng} / \mathrm{mL}$ fibroblast growth factor, (FGF, Millipore, Billerica, USA) and $50 \mathrm{ng} / \mathrm{mL}$ insulin-like growth factor (IGF, Sigma) were also added. The media composition has previously been used to culture inner ear derived cells from both mice and humans $(12,13)$.

Supplemented Neurobasal media (Gibco) contains B27 supplement (1x, Gibco), 2 mM Lglutamine (Gibco) and $0.04 \%$ gentamicin. Complete Neurobasal media also contains $20 \mathrm{ng} / \mathrm{mL}$ BDNF (R\&D Systems), 20 ng/mL NT-3 (R\&D Systems) and $20 \mathrm{ng} / \mathrm{mL}$ glial-derived neurotrophic factor (GDNF, R\&D Systems). Media has previously been used for primary human spiral ganglion culture (5).

\section{Human superior vestibular ganglion preparation}

Study on human materials was approved by the local ethics committee (no. 99398, 22/9 1999, cont., 2003, Dnr. 2013/190). Studies adhered to the rules of the Helsinki declaration. The superior vestibular nerve housing the SVG within the internal acoustic meatus is normally removed and discarded during translabyrinthine surgical removal of acoustic neuroma. Tissue was instead retrieved and immediately placed in Leibovitz's L-15 Medium (Gibco) in the operating room. Whilst submerged in media tissue was distributed into smaller pieces under dissection microscope (V20 stereo-microscope, Zeiss, Germany) (Figure 1).

Pieces containing the ganglion were washed three times in phosphate buffered saline (PBS, Gibco) and then either directly encapsulated in a gel or digested for $10 \mathrm{~min}$ in $0.25 \%$ Trypsin/EDTA. Trypsin was inactivated with DMEM (Gibco) containing $10 \%$ serum (Gibco). After centrifugation the supernatant was removed and cooled gel suspension was added to the digested tissue. For explant cultures Matrigel ${ }^{\mathrm{TM}}$ (Growth factor reduced Matrigel ${ }^{\mathrm{TM}}$, BD Biosciences, San Jose, USA) was mixed 1:1 with complete DMEM/F12; final Matrigel ${ }^{\mathrm{TM}}$ concentration was $3.9 \mathrm{mg} / \mathrm{mL}$. The gel suspension was prepared on ice. 8 well chambered slides 


\section{3-D culture of Human Vestibular Nerve}

113 were used where the piece of tissue was placed centrally and covered by $100 \mu \mathrm{L}$ of gel

114 suspension. After the gel had set for $30 \mathrm{~min}$ at $37^{\circ} \mathrm{C}$ and $5 \% \mathrm{CO}_{2}, 300 \mu \mathrm{L}$ of complete

115 DMEM/F12 media was added. After 24 hours the media was replaced with complete Neurobasal

116 media to stimulate neural outgrowth. Media was replaced every 2 - 3 days. SVG were obtained

117 from three patients and divided into 9 explants which were cultured for up to 8 days in the

118 described conditions before being fixated for $30 \mathrm{~min}$ in $4 \%$ paraformaldehyde.

Studies of hNPC, guinea pig and mouse spiral ganglion

120

121

122

123

124

125

126

127

128

129

130

131

132

133

134

135

136

137

138

139

140

A commercially available (ENStem-A, Millipore) human neural progenitor cell line (hNPC) was cultured as previously described (14). Before gel encapsulation, hNPC were maintained in proliferating conditions in supplemented Neurobasal media to which $20 \mathrm{ng} / \mathrm{mL}$ basic fibroblast growth factor (FGF-2, Millipore) and $10 \mathrm{ng} / \mathrm{mL}$ of leukemia inhibitory factor (LIF, Millipore) was added. Having grown close to confluence, cells were dissociated, centrifuged and resuspended in complete Neurobasal media at a concentration of 3000 cells $/ \mu \mathrm{L}$. The gel was prepared on ice as a 1:1 mixture of cell suspension and Matrigel $^{\mathrm{TM}}$ with a final cell concentration of 1500 cells $/ \mu \mathrm{L}$. The gel suspension was distributed in 8 well chambered slides (Millipore) and placed at $37{ }^{\circ} \mathrm{C}$ incubator, $5 \% \mathrm{CO}_{2}$, for $30 \mathrm{~min}$ to cure. Complete Neurobasal media was then carefully added on top of the gel and slides returned to incubator, media was replaced every $2-3$ days and cultures were maintained for 14 days before fixation for $30 \mathrm{~min}$ in $4 \%$ paraformaldehyde solution at room temperature.

Animal tissue was used in parallel with human vestibular tissue as human tissue is limited. The capacity for rodent vestibular and SG explants to grow in gels has been previously demonstrated $(4,8)$ and a minimum amount of animal cochleae were used. The use of two guinea pigs was approved by the local ethics committee (C98/12). Mice were sacrificed in accordance with ethical consent (C346/11) by neighboring group and the temporal bones from four P9 mice were afterwards generously donated in accordance with supplements to the ethical consent. Temporal bones were dissected and the spiral ganglions (SG) were isolated. Explants were treated the same way as described for the SVG, but were not enzymatically digested. After $8-9$ days in culture the tissue was fixed for $30 \mathrm{~min}$ in $4 \%$ paraformaldehyde solution at room temperature. 


\section{3-D culture of Human Vestibular Nerve}

141

142

143

144

145

146

147

148

149

150

151

152

153

154

155

156

157

158

159

160

161

162

163

164

165

166

167

168

169

\section{Time-lapse video microscopy}

An inverted Nikon TE2000-E microscope (Nikon, Tokyo, Japan) equipped with $\mathrm{CO}_{2}$-incubator (Figure 1D) and Nikon Perfect Focus System controlled via NIS Elements software (version 4.04) was used for time-lapse video recordings. Pictures were taken every minute during $12-48$ hours. Movies were saved as raw .tiff data files and in a compacted avi-format played at 14 frames per second.

\section{Image processing}

Measurements were made using ImageJ 1.46r (NIH, Bethesda, USA) or Gradientech Tracking Tool (Gradientech, Uppsala, Sweden).

\section{Immunohistochemistry}

The cultured explants were fixated using 4\% paraformaldehyde (PFA) solution at room temperature for $30 \mathrm{~min}$, followed by washing with 1x PBS and where then either stained as a whole mount or embedded in Tissue-Tek O.C.T (Sakura, Tokyo, Japan). Embedded samples were snap frozen in liquid nitrogen and stored at $-70{ }^{\circ} \mathrm{C}$ until use. $6 \mu \mathrm{m}$ sections were sectioned using a cryotome and carried on SuperFrostPlus (Menzel, Brauschweig, Germany) slides. The slides were post-fixated with aceton prior staining.

Samples were washed in phosphate buffered saline (PBS, Gibco) for 3 x 5 min. $0.4 \%$ Triton X100 permeabilization buffer was applied for $30 \mathrm{~min}$ followed by 3 x $5 \mathrm{~min}$ washing in PBS. The slides were blocked using $2 \% \mathrm{BSA} / \mathrm{PBS}$ for $30 \mathrm{~min}$ at $37^{\circ} \mathrm{C}$. Primary antibodies (anti-Tuj1 041049 or MAB1637, Millipore, 1/200; anti-BDNF sc-33904 Santa Cruz, CA, USA 1/100; antiTrkB sc-12, Santa Cruz, 1/50; anti-Glial Fibrillary Acidic Protein (GFAP), Millipore, MAB360. 1/100) were dissolved in $2 \% \mathrm{BSA} / \mathrm{PBS}$, applied and incubated at $4{ }^{\circ} \mathrm{C}$. The primary antibodies were removed and samples washed $3 \times 5$ min in PBS before applying the secondary antibodies at 1/200 dilution (All secondary antibodies purchased from Invitrogen, Carlsbad, CA, USA. Alexa Fluor® 555: Goat anti-Mouse, A21422; Donkey anti-Goat, A21432. Alexa Fluor® 488: Goat Anti-Rabbit A11008; Donkey anti-Rabbit, A21206) which was dissolved in $2 \%$ BSA/PBS. Secondary antibodies were applied and incubated for 3 hours in room temperature. Another 3 x 5 min washing with PBS followed before samples were mounted with Vectashield Mounting Medium with DAPI (Vector Laboratories, CA, USA). 


\section{3-D culture of Human Vestibular Nerve}

170 An inverted three color $(358,461$ and $555 \mathrm{~nm})$ fluorescence microscope equipped with three laser

171 confocal system (C1, Nikon) was used for image capturing (TE2000-E, Nikon).

\section{Results}

\section{3-D culture of human superior vestibular ganglion}

174 Explants from all three patients grew when placed in Matrigel ${ }^{\mathrm{TM}}$ under the described culture

175 conditions. However, no obvious difference was observed between trypsin-digested tissue and

176 undigested tissue in terms of extent and type of outgrowth. Single protrusions from cells could be

177 observed outside the explants within 24 hours and nerve-like extensions could be seen after 48

178 hours in some cultures. Explants were maintained in culture for up to 8 days. The most extensive

179 neural sprouting and glia and fibroblast outgrowth was obtained from a larger explant (Figure 2).

180 Neurons were capable of dynamic growth in the matrix, different levels were visible by shifting

181 between focal planes in the gel or in TLVM as the growth cones could be seen migrating in and

182 out of focus. The growth cones that sprouted expanded inside the gel in a random manner, both

183 straight and in circular fashion without obvious decision making (Figure 3) as visualized through

184 TLVM (Supp Video 1). Growth cones displayed small vesicular protrusions that penetrated the

185 gel in several directions and then withdrew in all but one direction. The gel did not restrict axonal

186 expansion which was often followed by retraction and redirection (Supp Video 1). After 5 days in

187 culture the average velocity of growth cone migration was $18 \mu \mathrm{m} / \mathrm{h}$. After fixation, the presence

188 of nerves could be verified through whole mount staining of explants in gel as well as

189 cryosectioning of the explants (Figure 4). Inside explants large nerve-like cell bodies with axon-

190 line projections could be found, as well as axon-like fibers that could not be traced to cell-bodies

191 which all stained positive for neural marker Tuj1 and the BDNF-receptor TrkB (Figure 4). The

192 Tuj1 positive cell bodies and fibers were surrounded by smaller GFAP positive cells.

193 hNPC, guinea pig and neonatal mouse culture

194 hNPC formed sphere-like structures which sprouted Tuj1-positive axon-like projections and

195 contacted neighboring spheres (Figure 5 Figure 1A, arrow). Internally, these spheres also

196 contained Tuj1-positive axon-like structures. 3-D outgrowth could be visualized using confocal

197 laser microscopy but was mainly seen by shifting between focal planes in the fluorescent

198 microscope. In some instances, cells continued to proliferate within the gel and formed dense 


\section{3-D culture of Human Vestibular Nerve}

199 layers on surfaces at the bottom of the gel despite withdrawal of mitogens and added 200 neurotrophic stimulation for 14 days.

201 In animal explant cultures, initial protrusions were visible within 24 hours and glia cells migrated 202 into the surrounding gel within 48 hours of seeding. Nerve-like cells started to appear after $2-3$

203 days in culture. In the P9 mouse explant some SG neuron cell bodies migrated peripherally out 204 from the explants and into the gel within 4 days (Figure 6 arrows, inset).

205 Tuj1 staining of cryosectioned P9 mouse cochlear explants verified typical round type I spiral 206 ganglion cell bodies in the explants where neural extensions had been seen in vitro (Figure 8). In 207 sections of mouse cochlea, BDNF expressing type I cells were found (Figure 8, inset).

208 In guinea pig SG cultures Tuj1 positive extensions could be seen in the gel as well as neural cell 209 bodies at the border of whole mounted explant cultured for 5 days (Figure 7 A). Aligment of 210 schwann cell-like cells around nerve-like extensions could also be observed through TLVM 211 (Figure 7 B, Supp. Video 2).

\section{Discussion}

213 Here, for the first time, we describe a technique to harvest and culture adult human SVG in 3-D 214 allowing us to study primary nerves from the human auditory system. Part of the normal human 215 vestibular nerve can be taken out during surgery to eliminate vestibular schwannoma with a 216 translabyrinthine approach when tumor growth is limited to the medial portion of the internal 217 acoustic meatus. The human SVG consists of several small populations of neurons located near 218 the five different sensory organs in each vestibular organ. These ganglia lie near the fundus 219 region except the singular or posterior vestibular nerve that enters the meatus anteriorly and more 220 medially. As the ganglion is only a minor part of the nerve and not easily observable only some 221 explants contained the ganglion. Limitations in the method only allowed us determine if an 222 explant actually contained the ganglion after the specimen had been sectioned and stained.

223 Trypsinization was used as it could be advantageous to loosen up the epineurium sheet to 224 facilitate neural outgrowth but we found no connection between digestion and amount of 


\section{3-D culture of Human Vestibular Nerve}

225 outgrowth in neither human SVG cultures nor animal SG cultures. In the end outgrowth will be

226 determined by the content of each individual explant.

227 The Matrigel ${ }^{\mathrm{TM}}$ had a stimulating effect on neural growth along with the added neurotrophins, but

228 supporting cells lining neural cell bodies and axons will continue to provide stimulus throughout

229 the culture and will also shield neural cell bodies from external cues. Likely, the stimulating

230 effects were further boosted by residual growth factors in the MatrigelTM. As it is derived from

231 Engelbreth-Holm-Swarm (EHS) sarcoma cells it contains basement membrane proteins such as

232 collagens, laminin, and proteoglycans making it a suitable environment for cells, but it also

233 contains degradation enzymes, inhibitors and various growth factors making it unsuitable for

234 clinical applications. The unreliable performance of the hNPC is also, likely, a symptom of the

235 residual growth factors in the Matrigel ${ }^{\mathrm{TM}}$.

236 As an alternative, 3-D type-I-collagen gels have been used to study and maintain organization of

237 neural networks and neurons/glial cell interaction as well as functional synapses and CNS stem

238 cell differentiation, but these gels also contain cell- or tissue derived components (15-17) .

239 Modifying gels using synthetic laminin motif isoleucine-lysine-valine-alanine-valine (IKVAV)

240 (18) but also self-assembled IKVAV peptide amphiphiles have been shown to stimulate neural

241 differentiation and maturation (19) making IKVAV an interesting candidate to find fully

242 synthetic implantable matrixes suitable to close the gap between CI and SG.

244 TLVM gave detailed information of the dynamics of nerve GC expansion. Tracking neurons in

245 this manner could be used to study effects from guidance molecules and influence of electric

246 stimulation on GC. We have previously demonstrated effects of small guidance molecules using

247 TLVM in 2-D cell culture (20). The present model provides tools to study these influences in a

248 more in vivo-like 3-D model. The physical interaction between neurites and glial/Schwann cells

249 is also of interest since attempted regeneration includes the ensheathment of neurons by glial

250 cells to reach functional maturity. Using TLVM in guinea pig SG culture this behavior could be

251 observed, it was however not captured in human cultures.

252

253 Recent findings (8) showed that vestibular neurons could establish de novo contacts with hair

254 cells in an organotypic model using explants from rodent vestibular sensory epithelia and 
255

256

257

258

259

260

261

262

263

264

265

266

267

268

269

270

271

272

273

274

275

276

277

278

279

280

281

282

283

284

285

286

287

vestibular ganglions. Our study shows that adult human SVG also has an innate capacity to regenerate. Rodent SG responded similarly to SVG using our protocol indicating that human SG could carry a similar regenerative capacity. That would in turn mean that human SG could potentially reach and contact regenerated hair cells in the organ of Corti or grow towards a CI placed in the scala tympani. Future studies will show if this assumption is correct.

The present study shows that Matrigel ${ }^{\mathrm{TM}}$ can support human SVG outgrowth as well as SG in an animal model and promote neural differentiation in hNPC. Future studies will show if a fully synthetic defined gel can be found which combines the nerve stimulating environment of Matrigel $^{\mathrm{TM}}$ with distinctive and optimal physiological properties and efficacy.

\section{Conclusions}

This study shows for the first time that human inner ear neurons can be harvested during translabyrinthine surgery for removal of vestibular schwannoma and grown 3-D in a lamininbased matrix (Matrigel ${ }^{\mathrm{TM}}$ ). The SVG had an innate regenerative capacity and were able to resprout. Animal cochlear nerve tissue responded correspondingly suggesting that this in vitro model can be used in translational studies of human auditory nerve signaling.

Reference

1. Wilson BS. Toward better representations of sound with cochlear implants. Nat Med. 2013 10//print;19(10):1245-8.

2. Geers AE. Factors influencing spoken language outcomes in children following early cochlear implantation. Advances in oto-rhino-laryngology. 2006;64:50-65. PubMed PMID: 16891836.

3. Brors D, Aletsee C, Schwager K, Mlynski R, Hansen S, Schäfers M, et al. Interaction of spiral ganglion neuron processes with alloplastic materials in vitro. Hearing research. 2002 5//;167(1-2):11021. 4. Xie J, Pak K, Evans A, Kamgar-Parsi A, Fausti S, Mullen L, et al. Neurotrophins differentially stimulate the growth of cochlear neurites on collagen surfaces and in gels. Neural regeneration research. 2013;8(17):1541-50. PubMed PMID: 24459465. Pubmed Central PMCID: 3897172.

5. Rask-Andersen H, Boström M, Gerdin B, Kinnefors A, Nyberg G, Engstrand T, et al. Regeneration of human auditory nerve. In vitro/in video demonstration of neural progenitor cells in adult human and guinea pig spiral ganglion. Hearing research. 2005 5//;203(1-2):180-91.

6. Gaboyard S, Chabbert C, Travo C, Bancel F, Lehouelleur J, Yamauchi D, et al. Three-dimensional culture of newborn rat utricle using an extracellular matrix promotes formation of a cyst. Neuroscience. $2005 / / ; 133(1): 253-65$. 
7. Gaboyard S, Sans A, Lehouelleur J. Differential impact of hypergravity on maturating innervation in vestibular epithelia during rat development. Developmental Brain Research. 2003 6/12/;143(1):15-23. 8. Travo C, Gaboyard-Niay S, Chabbert C. Plasticity of Scarpa's ganglion neurons as a possible basis for functional restoration within vestibular endorgans. Frontiers in Neurology. 2012 2012-June-6;3. English.

9. Liu W, Glueckert R, Linthicum FH, Rieger G, Blumer M, Bitsche M, et al. Possible role of gap junction intercellular channels and connexin 43 in satellite glial cells (SGCs) for preservation of human spiral ganglion neurons : A comparative study with clinical implications. Cell and tissue research. 2014 Feb;355(2):267-78. PubMed PMID: 24241398. Pubmed Central PMCID: 3921454.

10. Abelló G, Khatri S, Giráldez F, Alsina B. Early regionalization of the otic placode and its regulation by the Notch signaling pathway. Mechanisms of Development. 2007 8//;124(7-8):631-45.

11. Ernfors P, Van De Water T, Loring J, Jaenisch R. Complementary roles of BDNF and NT-3 in vestibular and auditory development. Neuron. 1995 6//;14(6):1153-64.

12. Oshima K, Senn P, Heller S. Isolation of sphere-forming stem cells from the mouse inner ear. Methods in molecular biology. 2009;493:141-62. PubMed PMID: 18839346. Pubmed Central PMCID: 2861714.

13. Rask-Andersen H, Bostrom M, Gerdin B, Kinnefors A, Nyberg G, Engstrand T, et al. Regeneration of human auditory nerve. In vitro/in video demonstration of neural progenitor cells in adult human and guinea pig spiral ganglion. Hearing research. 2005 May;203(1-2):180-91. PubMed PMID: 15855043. 14. Edin F, Liu W, Boström M, Magnusson PU, Rask-Andersen H. Differentiation of human neural progenitor cell-derived spiral ganglion-like neurons: a time-lapse video study. Acta oto-laryngologica. 2014;134(5):441-7. PubMed PMID: 24702223.

15. Bergsteinsdottir K, Hashimoto Y, Brennan A, Mirsky R, Jessen KR. The Effect of Three Dimensional Collagen Type I Preparation on the Structural Organization of Guinea Pig Enteric Ganglia in Culture. Experimental cell research. 1993 11//;209(1):64-75.

16. O'Shaughnessy TJ, Lin HJ, Ma W. Functional synapse formation among rat cortical neurons grown on three-dimensional collagen gels. Neuroscience Letters. 2003 4/17/;340(3):169-72.

17. Ma W, Fitzgerald W, Liu QY, O'Shaughnessy TJ, Maric D, Lin HJ, et al. CNS stem and progenitor cell differentiation into functional neuronal circuits in three-dimensional collagen gels. Experimental Neurology. 2004 12//;190(2):276-88.

18. Hosseinkhani H, Hiraoka Y, Li C-H, Chen Y-R, Yu D-S, Hong P-D, et al. Engineering ThreeDimensional Collagen-IKVAV Matrix to Mimic Neural Microenvironment. ACS Chemical Neuroscience. 2013 2013/08/21;4(8):1229-35.

19. Wu B, Zheng Q, Wu Y, Guo X, Zou Z. Effect of IKVAV peptide nanofiber on proliferation, adhesion and differentiation into neurocytes of bone marrow stromal cells. J Huazhong Univ Sci Technol [Med Sci]. 2010 2010/04/01;30(2):178-82. English.

20. Anderson M, Bostrom M, Pfaller K, Glueckert R, Schrott-Fischer A, Gerdin B, et al. Structure and locomotion of adult in vitro regenerated spiral ganglion growth cones-- a study using video microscopy and SEM. Hearing research. 2006 May;215(1-2):97-107. PubMed PMID: 16684592. 


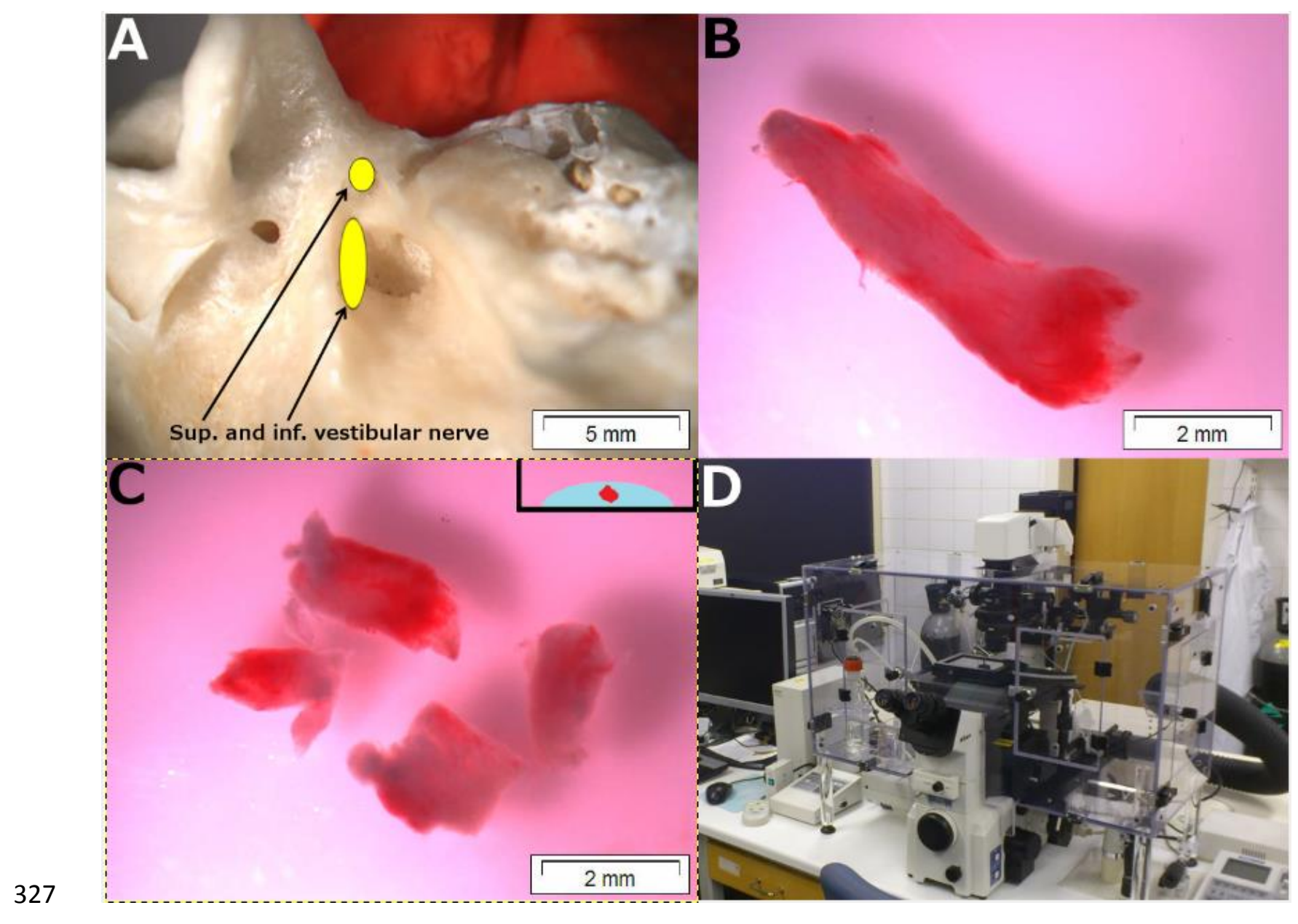

328 Figure 1 Dissection of human SVG A) Micro-dissected left human temporal bone showing the 329 location of the superior and inferior vestibular nerves. B) The vestibular nerve can be removed at 330 vestibular schwannoma surgery when the lateral aspect of the fundus is tumor-free. C) The nerve 331 was cut into smaller pieces before Matrigel ${ }^{\mathrm{TM}}$ encapsulation. Inset represents culture conditions 332 with tissue placed inside gel drop. D) Inverted microscope Nikon TE2000-E equipped with 333 fluorescence and confocal laser and digital camera used for time-lapse video recordings. 


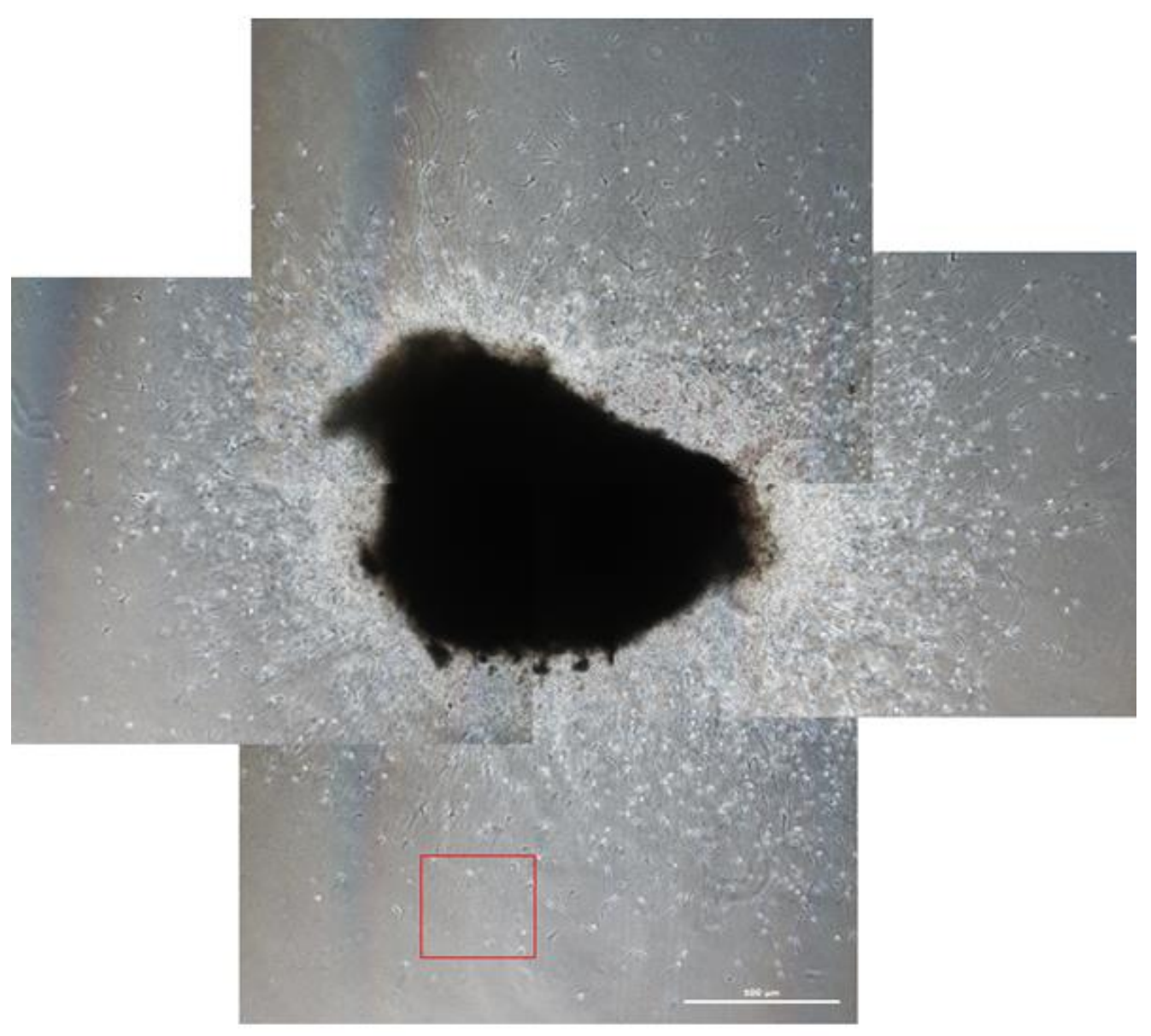

335 Figure 2 Human SVG explant culture. 3-D gel culture of trypsinized explant from human SVG 336 cultured for 6 days in Matrigel ${ }^{\mathrm{TM}}$. Glial cells expand peripherally but also neurites with typical 337 GCs. Red square indicates area filmed with TLVM (Supp. Video 1) and a single phase contrast 338 image from the TLVM is shown in figure 3. Scale bar is $500 \mu \mathrm{m}$. 


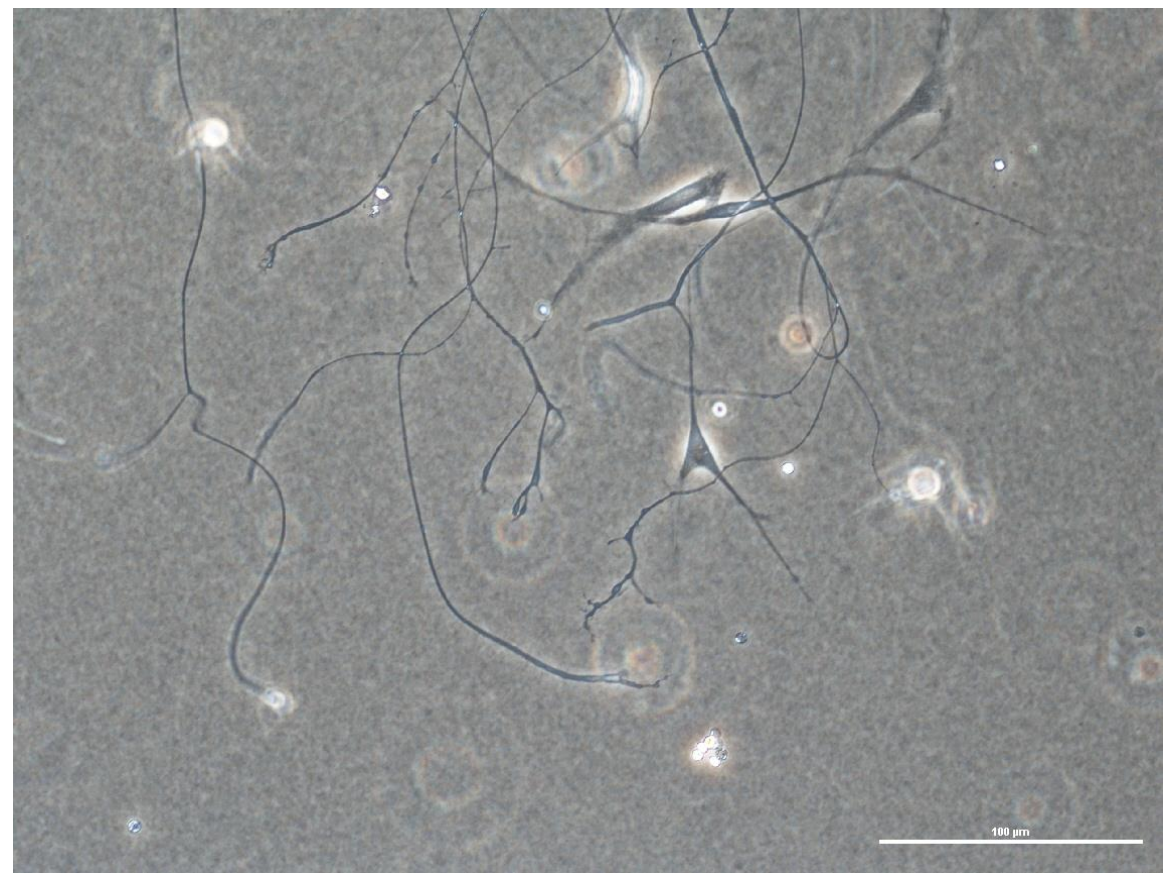

340 Figure 3 Neural outgrowth from human SVG in Matrigel $^{\text {TM }}$. Single phase contrast image 341 taken from TLVM (Supp. Video 1) presenting expanding human vestibular neurites from 342 trypsinized SVG in Matrigel ${ }^{\mathrm{TM}}$ after 6 days in culture showing the pattern of migration of the 343 growth cones in a 3-D gel. Picture was taken inside red square in Figure 2. Growth cones 344 migrated randomly, radiating out from the explant. Scale bar $100 \mu \mathrm{m}$. 


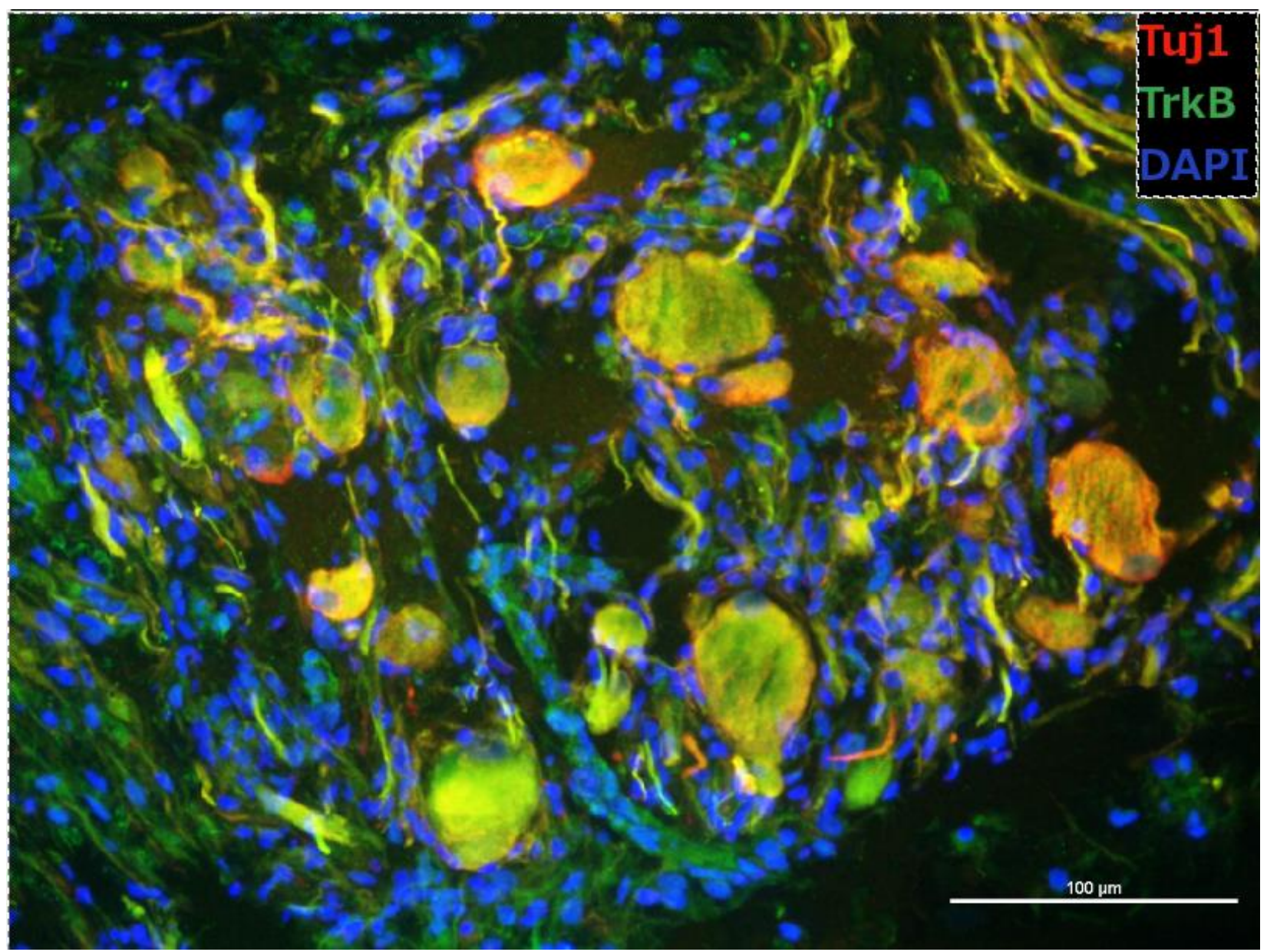

346 Figure 4 Cryosectioning and immunostaining of human SVG. Immunofluorescent image of 347 cryosectioned human SVG explant seen in Figure 2 fixated after 8 days of culture in Matrigel ${ }^{\mathrm{TM}}$. 348 Vestibular neurons are comparatively large and co-express Tuj1 (red) and TrkB (green). Scale 349 bar $100 \mu \mathrm{m}$. 


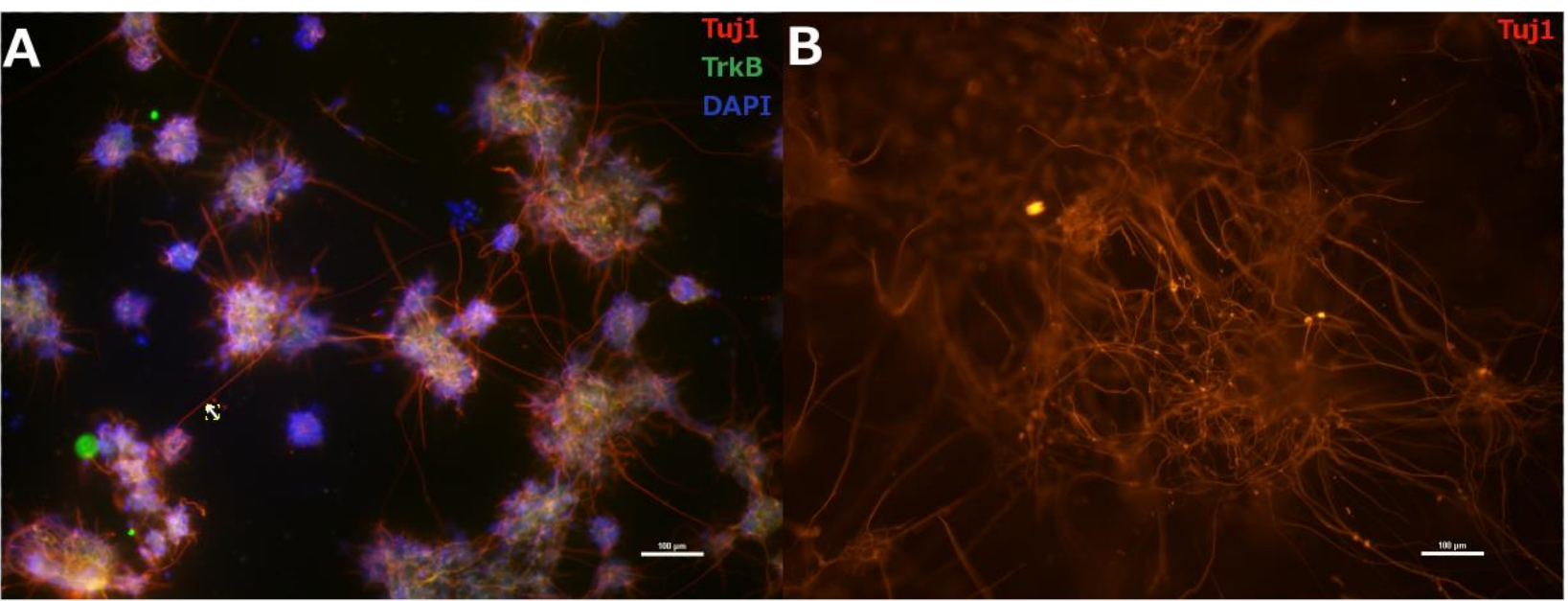

351 Figure 5 Differentiation of hNPC in Matrigel $^{\text {TM }}$. hNPCs were differentiated for 14 days

352 suspended in Matrigel ${ }^{\mathrm{TM}}$. A) Cells formed sphere-like structures containing Tuj1-positive (red)

353 neurons, the sphere-like structures also stained positive for TrkB (green). Nuclei were stained

354 with DAPI (blue). B) Neurons expanded heavily in the gel forming complex Tuj1-positive (red)

355 networks. Scale bars $100 \mu \mathrm{m}$. 


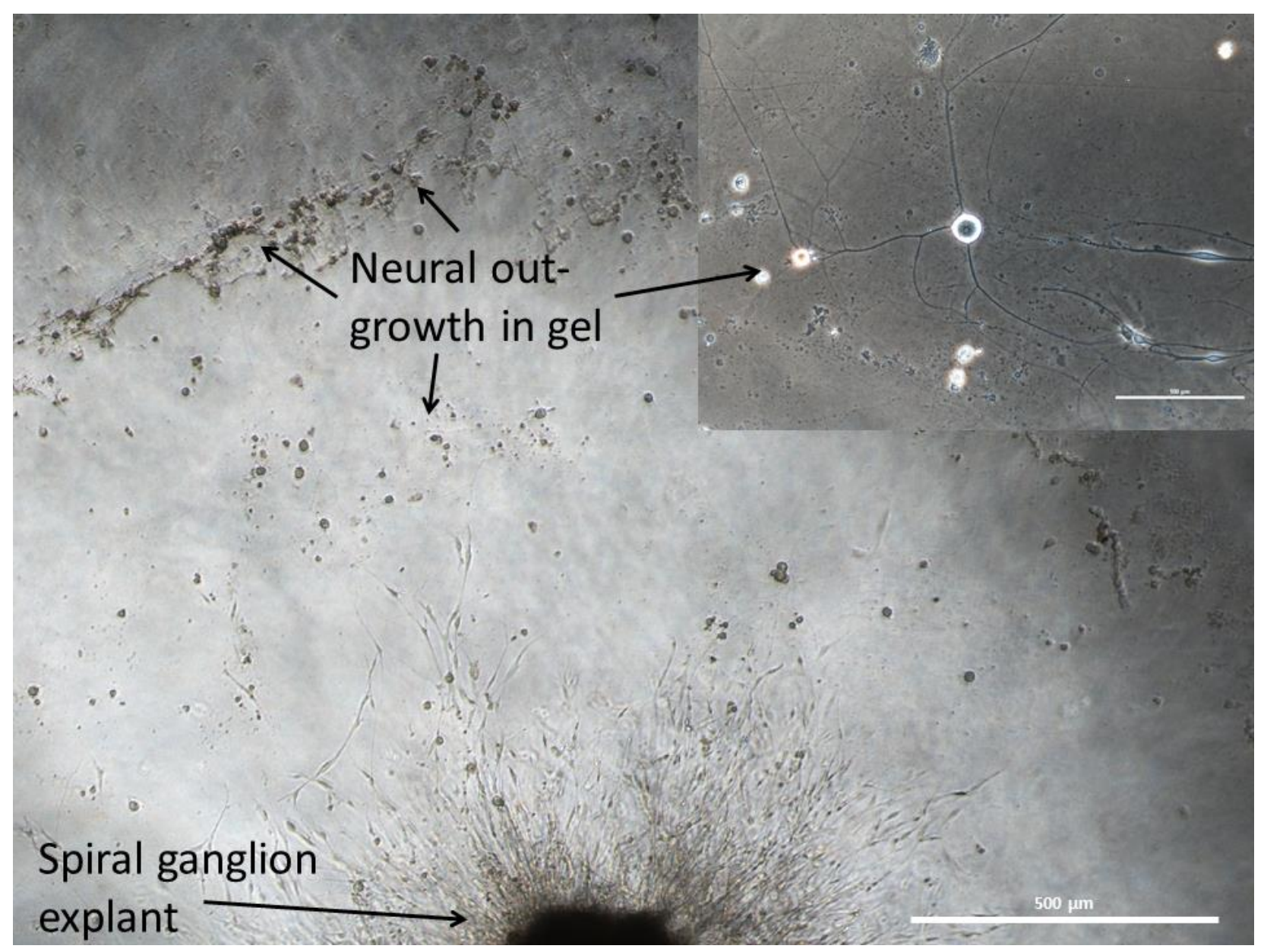

357 Figure 6 Culturing neonatal mice explants in Matrigel $^{\mathbf{T M}}$. Cochlear explants from neonatal 358 mice (P9) were placed in Matrigel $^{\mathrm{TM}}$ and treated as the human SVG. Glia cells and nerve soma 359 have migrated peripherally in the gel within 4 days. Scale bar $500 \mu \mathrm{m}$. Inset: Some nerve cell 360 nuclei (bodies) translocate into the surrounding gel along the projecting axon. These neurons 361 ended up as solitary cells in the gel adjacent to the explant. Scale bar $100 \mu \mathrm{m}$. 


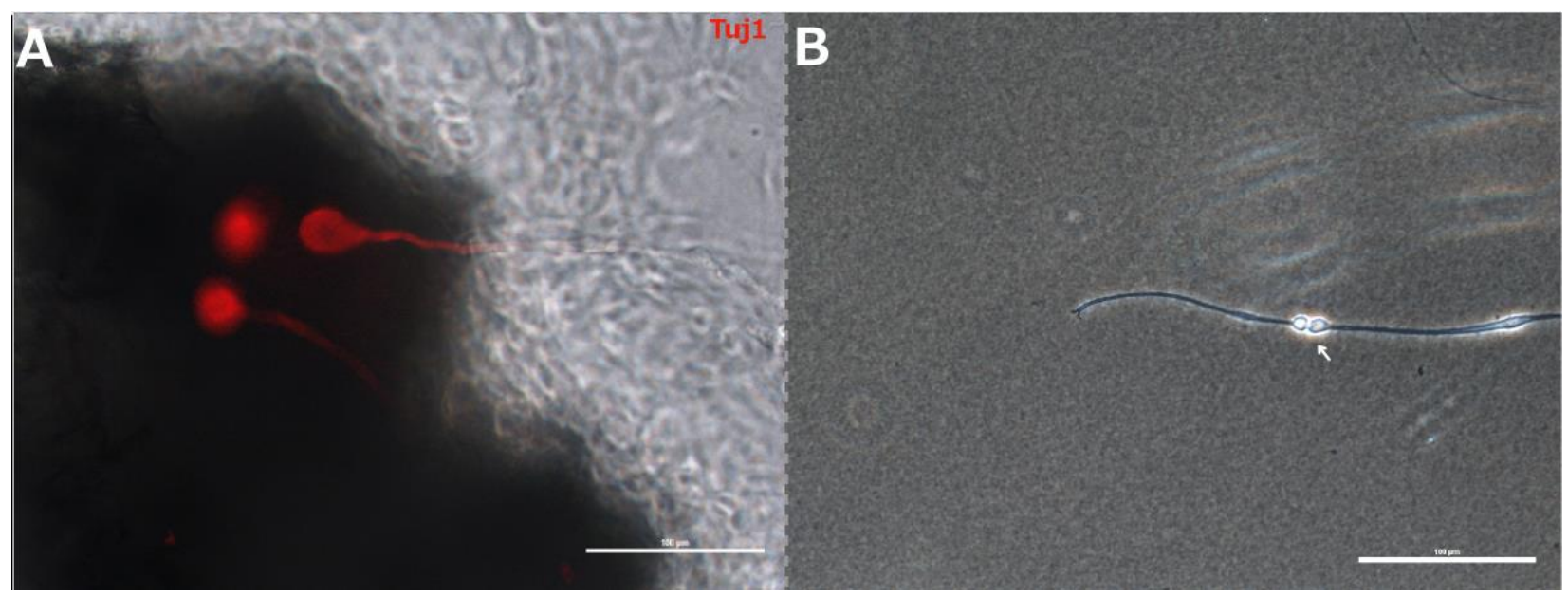

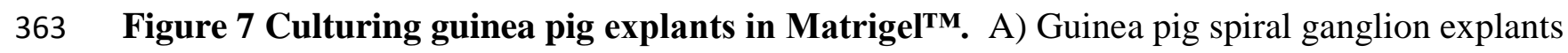

364 were cultured for 5 days before whole mount fixation and staining. Picture shows merged 365 immunofluorescent Tuj1-positive (red) stain and phase contrast image of three spiral ganglion 366 neurons found at the border between explant and gel at the bottom of the explant. B) Single 367 image taken from TLVM of a guinea pig SG neuron. Growth cone expands in the Matrigel 368 from right to left. Schwann cells align the neurite and migrate in the same direction. Arrow 369 indicates Schwann cell undergoing mitosis (Supp. Video 2). Scale bars $100 \mu \mathrm{m}$. 


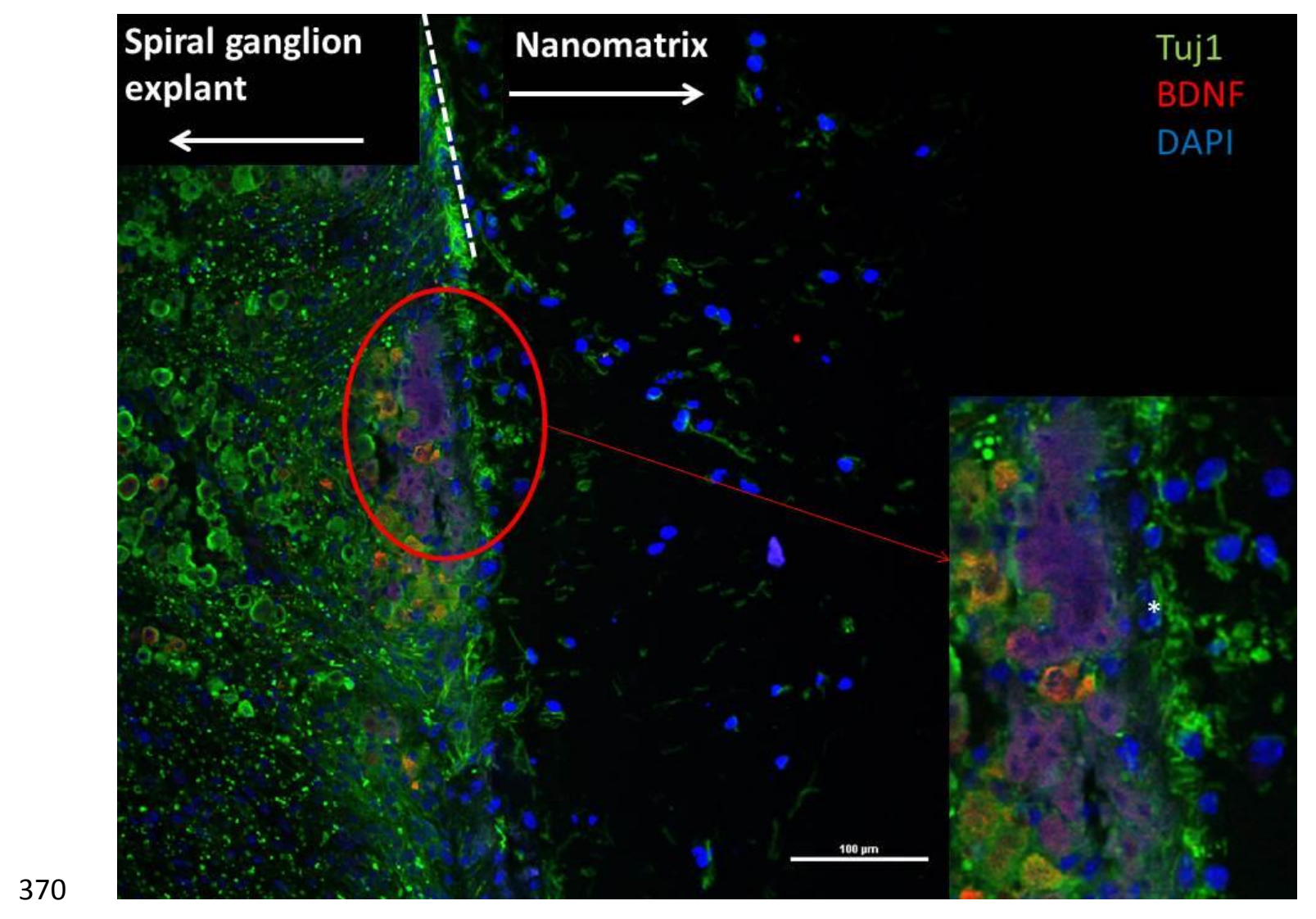

371 Figure 8 Cryosectioning and immunostaining of neonatal mice explants. Immunofluorescent

372 image showing a cochlear explant from neonatal mouse (P9) cryosectioned after 7 days in

373 culture. The spiral ganglion neurons are Tuj1-positive (green) and some also express BDNF

374 (red). Inset: Tuj1-positive fibers are seen to project from the tissue explants into surrounding

375 gel*. Nuclei were stained with DAPI (blue). Scale bar $100 \mu \mathrm{m}$. 